\title{
Identification of an HLA-A*0201-restricted cytotoxic T lymphocyte epitope from the lung carcinoma antigen, Lengsin
}

\author{
MUNEHIDE NAKATSUGAWA ${ }^{1}$, KAZUTAKA HORIE ${ }^{1}$, TOSHIAKI YOSHIKAWA ${ }^{1}$, MANAMI SHIMOMURA ${ }^{1}$, \\ YAMATO KIKUCHI $^{1}$, NORIKO SAKEMURA ${ }^{1}$, SHIRO SUZUKI $^{1}$, DAISUKE NOBUOKA ${ }^{1}$, \\ YOSHIHIKO HIROHASHI ${ }^{2}$, TOSHIHIKO TORIGOE ${ }^{2}$, KENJI HARADA $^{3}$, HIDEO TAKASU $^{3}$, \\ NORIYUKI SATO ${ }^{2}$ and TETSUYA NAKATSURA ${ }^{1}$

\begin{abstract}
${ }^{1}$ Section for Cancer Immunotherapy, Investigative Treatment Division, Research Center for Innovative Oncology, National Cancer Center Hospital East, 6-5-1 Kashiwanoha, Kashiwa, Chiba 277-8577; ${ }^{2}$ Department of Pathology, Sapporo Medical University School of Medicine, South-1, West-17, Chuo-ku, Sapporo 060-8556;

${ }^{3}$ Dainippon Sumitomo Pharma Co., Ltd., 6-8 Doshomachi 2-chome, Chuo-ku, Osaka 541-0045, Japan
\end{abstract}

Received April 27, 2011; Accepted June 2, 2011

DOI: 10.3892/ijo.2011.1089

\begin{abstract}
Lengsin is an eye lens protein with a glutamine synthetase domain. We previously identified this protein as a lung carcinoma antigen through cDNA microarray analysis. Lengsin protein is overexpressed irrespective of the histological type of lung carcinoma, but not in normal tissues other than the lens. Therefore, to significantly extend the use of Lengsinbased T-cell immunotherapies for the treatment of patients with lung carcinoma, we searched for HLA-A*0201-restricted epitopes from this protein by screening predicted Lengsin-derived candidate peptides for the induction of tumor-reactive CTLs. Four Lengsin-derived peptides were selected by computerized algorithm based on a permissive HLA-A*0201 binding motif, and were used to immunize HLA-A*0201 transgenic (HHD) mice. Two of the immunizing peptides, Lengsin(206215)(FIYDFCIFGV) and Lengsin(270-279)(FLPEFGISSA), induced peptide-specific cytotoxic T lymphocytes (CTLs) in HHD mice, and thus were used to stimulate human peripheral blood lymphocytes in vitro. Lengsin(206-215) and Lengsin (270-279) also induced human peptide-specific CTLs, and we were able to generate Lengsin(206-215)- and Lengsin(270279)-specific CTL clones. The Lengsin(270-279)-specific CTL clone specifically recognized peptide-pulsed T2 cells, COS-7 cells expressing HLA-A*0201 and Lengsin, and HLA-A*0201+/ Lengsin ${ }^{+}$lung carcinoma cells in an HLA-A*0201-restricted manner. On the other hand, the Lengsin(206-215)-specific CTL clone failed to recognize HLA-A $0201^{+} /$Lengsin $^{+}$target
\end{abstract}

Correspondence to: Dr Tetsuya Nakatsura, Section Head, Section for Cancer Immunotherapy, Investigative Treatment Division, Research Center for Innovative Oncology, National Cancer Center Hospital East, 6-5-1 Kashiwanoha, Kashiwa, Chiba 277-8577, Japan

E-mail: tnakatsu@east.ncc.go.jp

Key words: Lengsin, lung carcinoma antigen, cytotoxic T lymphocyte epitope, HLA-A*0201-restricted cells in the absence of cognate peptide. These results suggest that Lengsin(270-279) is naturally processed and presented by HLA-A*0201 molecules on the surface of lung carcinoma cells and may be a new target for antigen-specific T-cell immunotherapy against lung cancer.

\section{Introduction}

Lung cancer is the leading cause of cancer death in the world (1). Current chemotherapy and radiotherapy regimens provide a limited survival benefit and are often toxic as well as ineffective. Tumor antigen-specific T-cell immunotherapy is a promising new approach to cancer treatment that is more effective and less toxic. Recent studies have shown that the adoptive transfer of normal peripheral lymphocytes genetically modified by the insertion of tumor-reactive T-cell receptors (TCRs) can mediate in vivo complete regression in patients with metastatic melanoma $(2,3)$, and synovial cell sarcoma (4). Identification of naturally presented peptides derived from tumor-associated antigens on the surface of tumor cells, which can induce peptide-specific and tumor-reactive cytotoxic $\mathrm{CD}^{+} \mathrm{T}$ cells (CTLs), is required for antigen-specific T-cell immunotherapy including TCR gene transfer therapy as the therapeutic target. To date, many immunogenic CTL epitopes in the context of HLA-A*0201, which is the predominant subtype in most ethnic groups (5), have been identified by reverse immunology approach (6-8). In addition, $\mathrm{H}-2 \mathrm{D}^{\mathrm{b}-/ /}, \beta_{2} \mathrm{~m}^{--}$, HLA-A 0201 monochain transgenic (HHD) mouse is a useful animal model for assessing the ability of individual peptides to induce HLA-A*0201-restricted CTL response (9-15). Previously, we used genome-wide cDNA microarray analysis to identify a novel lung cancer antigen, Lengsin, as a potential target for immunotherapy (16). Lengsin protein is overexpressed irrespective of the histological type of lung carcinoma, but not in normal tissues other than the lens. Because the eye lens is an immune-privileged site $(17,18)$, similar to the case of the testis, Lengsin can be an attractive target for tumor antigen-specific immunotherapy, as with cancer-testis antigens. 
Table I. The affinity of Lengsin-derived peptides for HLA-A*0201 molecules.

\begin{tabular}{lclrr}
\hline Peptide (position) & Amino acid length & Peptide sequence & Binding score $^{\mathrm{a}}$ & $\%_{\text {MFI increase }}^{\mathrm{b}}$ \\
\hline Lengsin(149-158) & 10 & LMPELSTFRV & 2030 & 79 \\
Lengsin(206-215) & 10 & FIYDFCIFGV & 7699 & 65 \\
Lengsin(270-279) & 10 & FLPEFGISSA & 215 & 75 \\
Lengsin(347-355) & 9 & GLLKHSAAL & 103 & 44 \\
HIV-gag(77-85) & 9 & SLYNTYATL & 66 \\
\hline
\end{tabular}

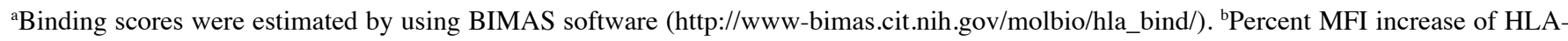
A*0201 molecules on T2 cells. Percent MFI increase $=($ MFI with sample peptide - MFI without peptide $) /$ MFI without peptide $x 100$. See the HLA-A*0201-binding assay in Materials and methods.

In the current study, we attempted to identify Lengsinderived CTL epitope via a reverse immunology approach. We studied four Lengsin-derived peptides with the HLA-A*0201 binding motif selected by HLA-peptide binding predictions of the Bioinformatics and Molecular Analysis Section (BIMAS) program and evaluated their ability to provoke peptide-specific CTL responses in HLA-A*0201 transgenic (HHD) mice, and then we induced and generated peptide-specific CTL clones from peripheral blood lymphocytes of HLA-A* $0201^{+}$ healthy donors. We report here that the Lengsin(270-279) (FLPEFGISSA)-specific human CTL clones specifically recognize peptide-pulsed T2 cells, COS-7 transfectants expressing HLA-A 0201 and Lengsin, and HLA-A*0201+/Lengsin ${ }^{+}$tumor cells in an HLA-A*0201-restricted manner.

\section{Materials and methods}

Animals. HLA-A*0201-transgenic HHD mice were described previously (9). H-2 $\mathrm{D}^{\mathrm{b}-/} \beta_{2} \mathrm{~m}^{-/-}$double knockout mice introduced with human $\beta_{2}$ m-HLA-A2.1 ( $\left.\alpha 1 \alpha 2\right)-H-2 D^{b}$ ( $\alpha 3$ transmembrane cytoplasmic) (HHD) monochain construct gene were generated at the Département SIDA-Rétrovirus, Unité d'Immunité Cellulaire Antivirale, Institut Pasteur, France and kindly provided by Dr F.A. Lemonnier. The mice were kept under specific pathogen-free conditions. Mouse experiments were approved by the Animal Research Committee of National Cancer Center Hospital East.

Peptides and plasmids. Human Lengsin-derived peptides (purity $>90 \%$ ) sharing the amino acid sequences with mouse Lengsin and carrying binding motifs for HLA-A*0201-encoded molecules, were identified using HLA-peptide binding predictions of the Bioinformatics and Molecular Analysis Section (BIMAS) program (http://bismas.dcrt.nih.gov/molbio/hla bind/index.html). We purchased a total of four Lengsin-derived peptides carrying HLA-A*0201 binding motifs (Table I) from GeneWorld (Tokyo, Japan). HIV-gag (77-85)(SLYNTYATL) peptide was used as an irrelevant peptide in murine and human CTL assays. Full-length Lengsin cDNA was obtained from a human lung carcinoma cell line and subcloned into pcDNA3.1 vector (Invitrogen) as described previously (16). Expression vector pcDNA3.1 containing the HLA-A*0201 cDNA was provided by Riken BRC (19). The HLA-A*0201 cDNA was subcloned into pIRES-puro vector (Clontech) for stable transfection.
Cell lines. Human liver cancer cell line SK-Hep-1 (HLA$\mathrm{A}^{*} 0201^{+} /$Lengsin $^{-}$) and simian COS-7 (HLA-A*0201\%/Lengsin ${ }^{-}$) were obtained from the American Type Culture Collection (Manassas, VA, USA). Human lung carcinoma cell line 1-87 (HLA-A*0201-/Lengsin ${ }^{+}$) was obtained from the Cell Resource Center for Biomedical Research, Tohoku University (Sendai, Japan). T2 is a lymphoblastoid cell line that lacks TAP function and has HLA-A*0201 molecules that can be easily loaded with exogenous peptides. 1-87 and SK-Hep-1 were cultured in DMEM supplemented with $10 \%$ heat-inactivated fetal bovine serum (FBS, Gibco). T2 was cultured in RPMI supplemented with $10 \%$ heat-inactivated FBS. The 1-87-A0201 cell line $\left(\right.$ HLA-A ${ }^{*} 0201^{+} /$Lengsin $^{+}$) was obtained by stable transfection of 1-87 with HLA-A*0201 cDNA, and the SK-Hep-1-Lengsin $\left(\right.$ HLA-A $0201^{+} /$Lengsin $^{+}$) cell line was obtained by stable transfection of SK-Hep-1 with full-length Lengsin cDNA using FuGene ${ }^{\circledR}$ HD (Roche Applied Science). 1-87-A0201 and SK-Hep-1-Lengsin was cultured in DMEM supplemented with $10 \%$ heat-inactivated FBS containing $1 \mu \mathrm{g} / \mathrm{ml}$ puromycin (Sigma-Aldrich) and $1 \mathrm{mg} / \mathrm{ml} \mathrm{G} 418$ (Gibco), respectively. In addition, COS-7 cells transiently expressing HLA-A*0201 and/ or full-length Lengsin as the target cells were generated by cotransfection with pCDNA3.1 vector encoding these proteins using Lipofectamine 2000 (Invitrogen).

$H L A-A^{*} 0201-b i n d i n g$ assay. To determine the binding ability of the predicted peptides to HLA-A*0201 molecules, an in vitro cellular binding assay was performed as previously reported (20). Briefly, after incubation of T2 cells in culture medium at $26^{\circ} \mathrm{C}$ for $18 \mathrm{~h}$, cells $\left(2 \times 10^{5}\right)$ were washed with PBS and suspended in $1 \mathrm{ml}$ of Opti-MEM ${ }^{\circledR}$ (Invitrogen) with or without $100 \mu \mathrm{g}$ of peptide, followed by incubation at $26^{\circ} \mathrm{C}$ for $3 \mathrm{~h}$ and then at $37^{\circ} \mathrm{C}$ for $3 \mathrm{~h}$. After washing with PBS, HLA-A*0201 expression was measured by flow cytometry using FITCconjugated HLA-A2-specific monoclonal antibody (mAb) (BB7.2; BioLegend), and mean fluorescence intensity (MFI) was recorded. Percent MFI increase was calculated as follows: Percent MFI increase $=($ MFI with the given peptide - MFI without peptide)/(MFI without peptide) x 100 .

Induction of Lengsin-derived peptide-specific CTLs in HHD mice. In vivo immunization of mice and in vitro stimulation of primed spleen cells were performed as previously described (12). Briefly, bone marrow (BM) cells $\left(2 \times 10^{6}\right)$ from HHD mice 
were cultured in RPMI-1640 supplemented with 10\% FBS, together with granulocyte macrophage colony-stimulating factor $(5 \mathrm{ng} / \mathrm{ml})$ and $2-\mathrm{ME}(0.8 \mathrm{ng} / \mathrm{ml})$ for 7 days in $10-\mathrm{cm}$ plastic dishes, and these bone-marrow-derived dendritic cells (BM-DCs) were pulsed with a mixture of the four Lengsinderived peptides carrying HLA-A*0201 binding motifs $(1 \mu \mathrm{M}$ for each peptide) at $37^{\circ} \mathrm{C}$ for $2 \mathrm{~h}$. We primed the HHD mice with this syngeneic BM-DC vaccine $\left(5 \times 10^{5} / \mathrm{mouse}\right)$ into the peritoneal cavity once a week for two weeks. Seven days after the last immunization, the spleens were collected and CD4-negative spleen cells were isolated by negative selection with anti-CD4 microbeads (Miltenyi Biotec, Bergisch Gladbach, Germany) to exclude any non-specific IFN- $\gamma$ production by $\mathrm{CD} 4^{+}$spleen cells cocultured with the BM-DCs. The CD4-negative spleen cells $\left(2 \times 10^{6} /\right.$ well) were restimulated with syngeneic BM-DCs $\left(2 \times 10^{5} /\right.$ well $)$ pulsed once with the mixture of peptides in vitro. Six days later, the frequency of cells producing IFN- $\gamma / 1 \times 10^{5} \mathrm{CD} 4-$ negative spleen cells upon stimulation with syngeneic BM-DCs $\left(5 \times 10^{4} /\right.$ well) pulsed with each peptide or the irrelevant peptide, was evaluated in an enzymelinked immunospot (ELISPOT) assay as described below.

Generation of Lengsin peptide-specific $C D 8^{+} T$ cell clones from human PBMCs. This study was approved by the Ethics Committee of the National Cancer Center, and conforms to the ethical guidelines of the Declaration of Helsinki (1995). CTL clones were generated using the methods described previously with some modifications (21). Blood samples were collected from HLA-A $0201^{+}$healthy donors, after informed consent was obtained. Peripheral blood mononuclear cells (PBMCs) were isolated from whole blood by Ficoll-Paque (GE Healthcare) density gradient centrifugation. PBMCs were cultured $\left(5 \times 10^{6}\right.$ cells $/$ well $)$ with 1 or $0.1 \mu \mathrm{g} / \mathrm{ml}$ Lengsin-derived peptide in AIM-V ${ }^{\circledR}$ medium supplemented with $10 \%$ human AB serum, $10 \mathrm{U} / \mathrm{ml}$ recombinant human interleukin (rhIL)-2 (Chiron, Emeryville, CA, USA) once a week for two or three weeks and supplemented with $10 \mathrm{U} / \mathrm{ml}$ rhIL-2 between stimulations. Seven days after the last stimulation, $\mathrm{CD}^{+} \mathrm{T}$ cells were isolated using a CD8 T-cell isolation kit (Miltenyi Biotec) and plated at 3,1 and 0.3 cells/well in 96-well round-bottom plates with $5 \mu \mathrm{g} / \mathrm{ml}$ PHA-P (Wako, Tokyo, Japan), $200 \mathrm{U} / \mathrm{ml}$ rhIL-2 and $8 \times 10^{4}$ cells/well allogeneic irradiated (100 Gy) PBMCs. Proliferating $\mathrm{T}$ cell clones were screened for peptide-specific IFN- $\gamma$ production by ELISPOT assay using T2 cells pulsed with or without the immunizing peptide.

Enzyme-linked immunosorbent spot(ELISPOT) assay. Specific IFN- $\gamma$ secretion of murine and human CTLs in response to stimulator cells was assayed using the IFN- $\gamma$ ELISPOT Kit (BD Biosciences) according to the manufacturer's instructions. Stimulator cells were pulsed with or without peptide for $1 \mathrm{~h}$ at $37^{\circ} \mathrm{C}$ and then washed thrice. Responder cells $\left(5 \times 10^{4} /\right.$ well) were incubated with stimulator cells for $20 \mathrm{~h}$. In blocking experiments, peptide-pulsed T2 or tumor cells were preincubated with HLA-A2-specific mAb (BB7.2) or isotype control mAb (mIgG2b; BioLegend) for $1 \mathrm{~h}$. The resulting spots were counted using an ELIPHOTO counter (Minerva Tech, Tokyo, Japan).

Cytotoxicity assay. To measure cytolytic activity of the CTL clones, calcein AM release-based cytotoxic cell assay was

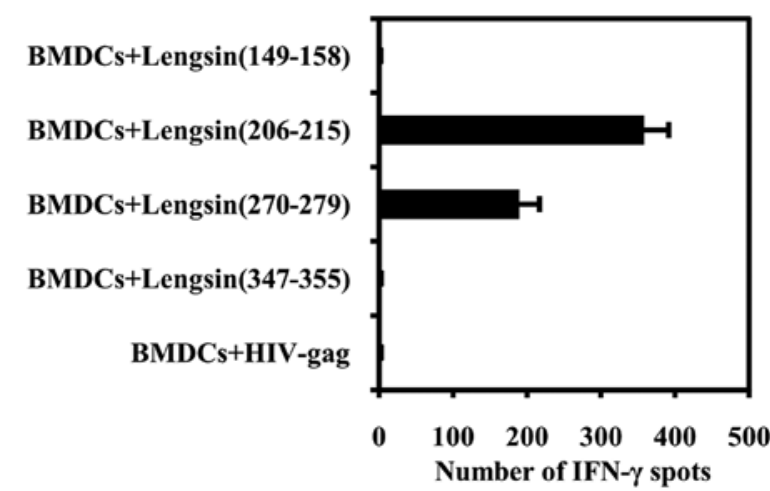

Figure 1. Immunogenicity of Lengsin-derived peptides in HHD mice. CD4negative spleen cells from HHD mice vaccinated with a mixture of four peptides were restimulated once in vitro with BM-DCs loaded with the mixture of peptides. CTLs were tested for specificity of each peptide by IFN- $\gamma$ ELISPOT assay. CTLs were stimulated with BM-DCs pulsed with $1 \mu \mathrm{M}$ of each peptide or an irrelevant peptide (HIV-gag). Results are expressed as the mean $\pm \mathrm{SD}$. A representative of three experiments is shown.

performed as described previously $(22,23)$. Briefly, $2 \times 10^{6}$ target cells were labeled with green fluorescent probe calcein AM at $37^{\circ} \mathrm{C}$ for $30 \mathrm{~min}$ and washed thrice. Target cells were pulsed with or without peptide for $1 \mathrm{~h}$ at $37^{\circ} \mathrm{C}$ and washed thrice. Effector cells were incubated with $1 \times 10^{4}$ target cells for $4 \mathrm{~h}$ at the indicated effector-to-target (E:T) ratios. The fluorescence emitted by target cells was measured using a Terascan system (Minerva Tech, Tokyo, Japan) before and after coincubation with effector cells. Percentage of specific lysis was determined as: (experimental release - spontaneous release)/(maximal release - spontaneous release) x 100 .

\section{Results}

Identification of Lengsin-derived peptide binding to HLA-A*0201 molecules. As the candidates of HLA-A*0201restricted and human Lengsin-derived CTL epitopes, we selected four peptides having high predicted HLA-A*0201binding scores calculated using the BIMAS software program, and we evaluated their binding ability to HLA-A*0201 molecules (Table I). All four peptides were able to bind to HLA-A*0201 molecules.

Induction of CTL response against the Lengsin-derived peptides in HHD mice. To evaluate the immunogenic potential of the four predicted HLA-A*0201-binding peptides derived from Lengsin, we immunized HHD mice with BM-DCs pulsed with a mixture of the four peptides. The results of ELISPOT assays revealed that the CD4-negative spleen cells stimulated in vitro with BM-DCs efficiently produced IFN- $\gamma$ when pulsed with the Lengsin(206-215) or Lengsin(270-279) but not with Lengsin(149-158), Lengsin(347-355), or HIV-gag peptides (Fig. 1). Similar results were obtained in three independent experiments. These results suggest that Lengsin(206-215) and Lengsin(270-279) have immunogenic potential and are able to induce peptide-specific CTLs in HHD mice.

Generation of Lengsin-peptide-specific CTL clones from human PBMCs. Next, we assessed the capacity of Lengsin(206- 
A.

HD1

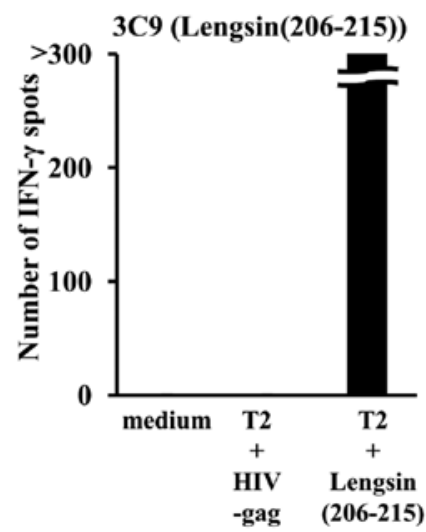

HD3

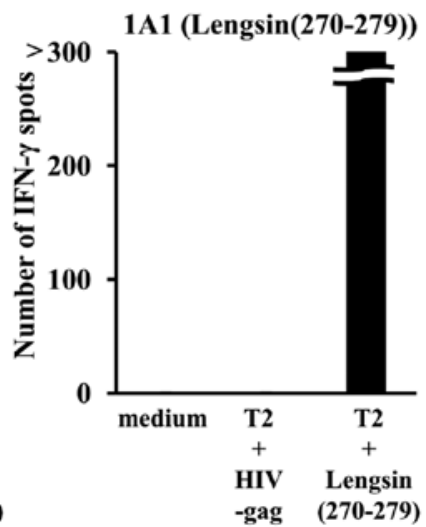

HD2

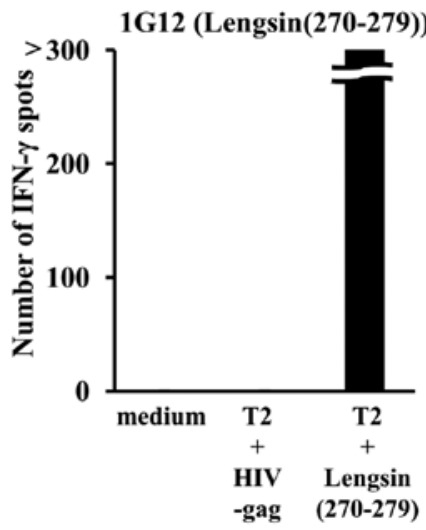

B.
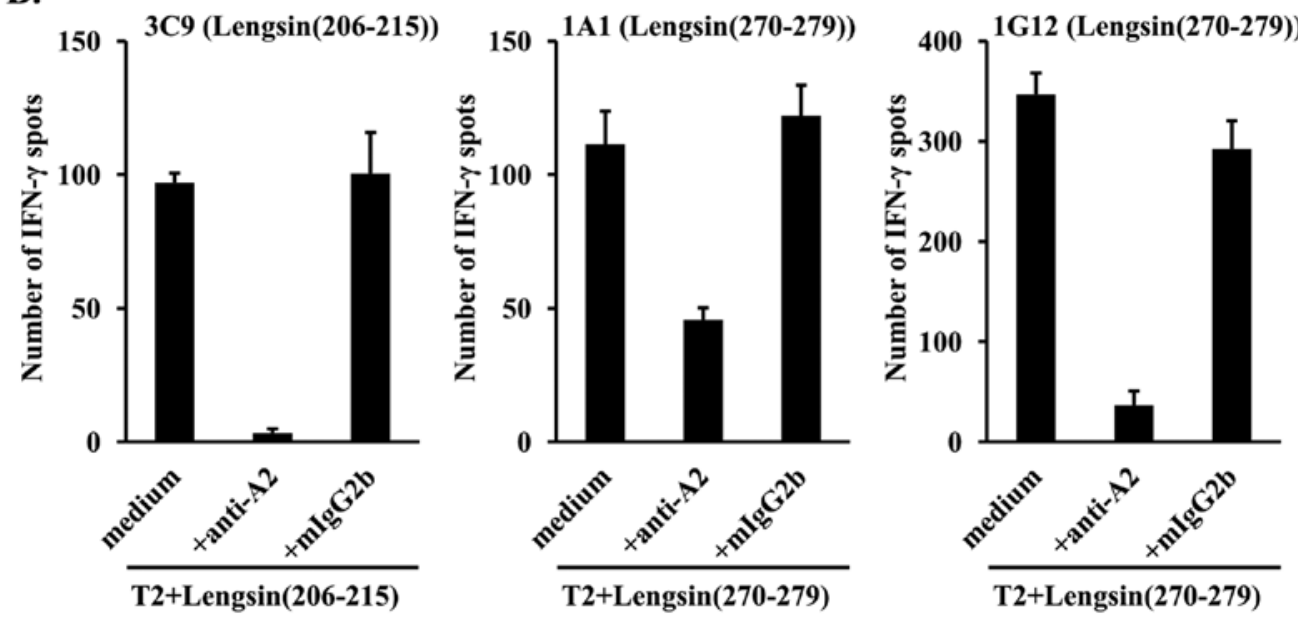

C.

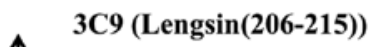

1A1 (Lengsin(270-279))

$1 \mathrm{G12}$ (Lengsin(270-279))
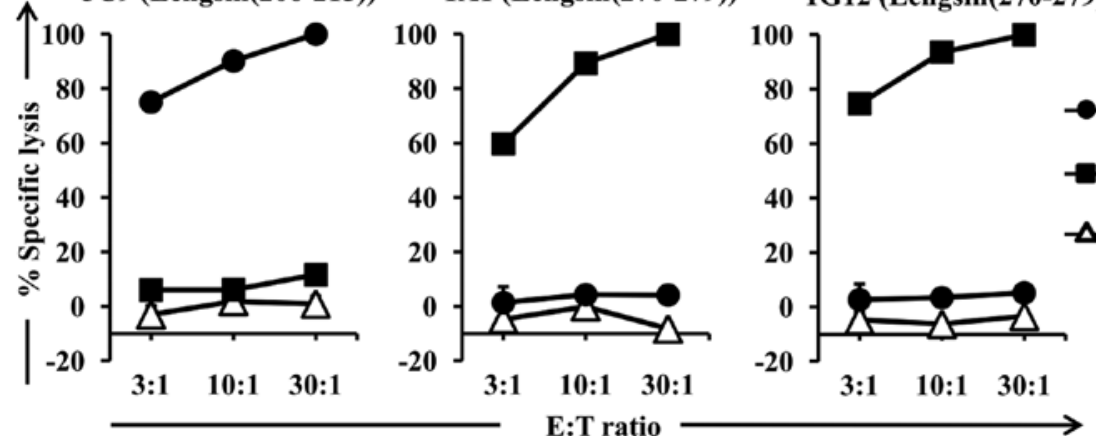

Figure 2. Recognition of peptide-pulsed T2 cells by human CTL clones. (A) Lengsin(206-215)-specific CTL clone 3C9 (1x104 cells) was stimulated with T2 cells pulsed with $1 \mu \mathrm{M}$ Lengsin(206-215) or HIV-gag peptide. Lengsin(270-279)-specific CTL clones $1 \mathrm{~A} 1$ and $1 \mathrm{G} 12$ (1x10 ${ }^{4}$ cells) were stimulated with T2 pulsed with $1 \mu \mathrm{M}$ Lengsin(270-279) or HIV-gag peptide. IFN- $\gamma$-producing CTLs were detected by IFN- $\gamma$ ELISPOT. (B) Blocking experiments were performed using HLA-A2-specific mAb (BB7.2) or isotype control mAb (mIgG2b). Each CTL clone $\left(1 \times 10^{3}\right.$ cells) was incubated with cognate peptide-pulsed T2 cells as stimulator cells. IFN- $\gamma$-producing CTLs were detected by IFN- $\gamma$ ELISPOT. (C) The cytolytic activity of Lengsin(206-215)-specific CTL clone 3C9 or Lengsin(270-279)-specific CTL clones 1A1 and 1G12 was assessed by cytotoxicity assay against T2 cells pulsed with $1 \mu \mathrm{M}$ Lengsin(206-215) (•), Lengsin(270-279) (-) or HIV-gag $(\Delta)$ peptide. Results are expressed as the mean \pm SD.

215) or Lengsin(270-279) to generate peptide-specific CTLs in vitro from human PBMCs of HLA-A $0201^{+}$healthy donors. CTLs were induced by two or three weekly in vitro stimulations with the Lengsin(206-215) or Lengsin(270-279) and were subsequently cloned by limiting dilution. We obtained one CTL clone (clone 3C9 derived from healthy donor (HD)1), which was able to specifically recognize $\mathrm{T} 2$ cells pulsed with
Lengsin(206-215) but not T2 cells pulsed with irrelevant HIV-gag peptide, and two CTL clones (clone 1A1 derived from HD3 and clone 1G12 derived from HD2), which were able to specifically recognize T2 cells pulsed with Lengsin(270-279) but not T2 cells pulsed with HIV-gag peptide, assessed by IFN- $\gamma$ ELISPOT assay (Fig. 2A). These three CTL clones had a $\mathrm{CD}^{+}{ }^{+} \mathrm{CD} 8{ }^{+} \mathrm{CD} 4-$ phenotype determined by flow cytometric 


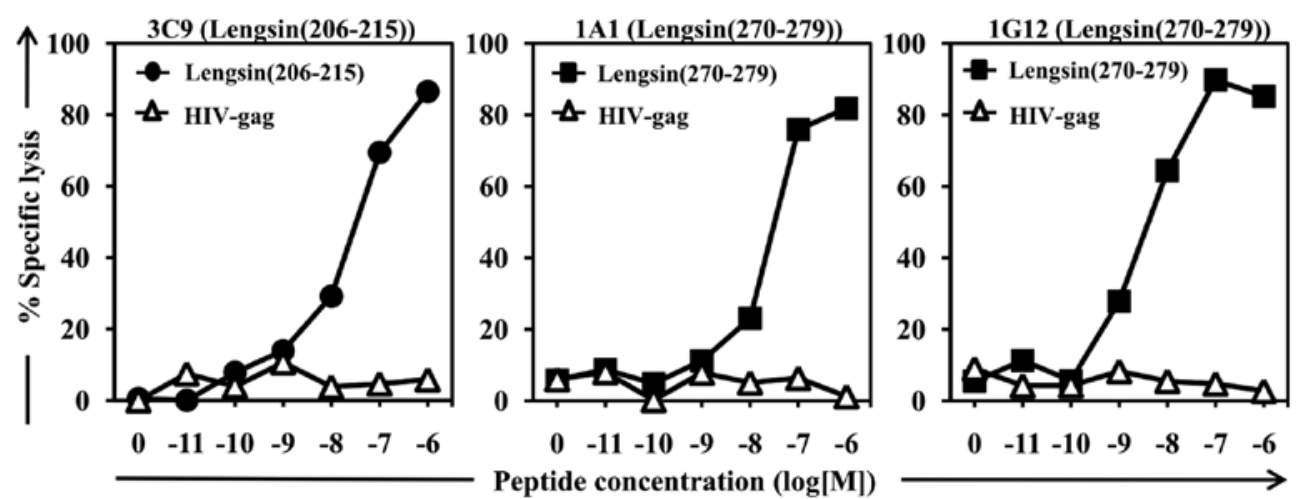

Figure 3. Functional avidity of CTL clones was determined by peptide titration experiments in cytotoxicity assay. Lengsin(206-215)-specific CTL clone 3C9 was incubated with titrated Lengsin(206-215) (•) or HIV-gag $(\Delta)$ peptide on T2 cells at an E:T ratio of 10:1. Lengsin(270-279)-specific CTL clones 1A1 and $1 \mathrm{G} 12$ were incubated with titrated Lengsin(270-279) (-) or HIV-gag ( $\Delta)$ peptide on T2 cells at an E:T ratio of 10:1. A representative of two experiments is shown.

analysis (data not shown). In addition, IFN- $\gamma$ production of CTL clones against cognate peptide-pulsed T2 was inhibited by HLA-A2-specific $\mathrm{mAb}$, but not isotype control $\mathrm{mAb}$, indicating that recognition by these CTL clones was HLA-A2 restricted (Fig. 2B). Moreover, we evaluated the cytolytic activity of these CTL clones against cognate peptide-pulsed T2 cells (Fig. 2C). Lengsin(206-215)-specific CTL clone 3C9 could specifically lyse Lengsin(206-215)-pulsed T2 cells but not Lengsin(270-279)- or HIV-gag peptide-pulsed T2 cells, and Lengsin(270-279)-specific CTL clones 1A1 and 1G12 could specifically lyse Lengsin(270-279)-pulsed T2 cells but not Lengsin(206-215)- or HIV-gag peptide-pulsed T2 cells. These results suggest that Lengsin(206-215) or Lengsin (270-279) can induce peptide-specific CTLs from human PBMCs, and Lengsin(206-215)- or Lengsin(270-279)-specific CTL clones specifically recognize cognate peptide-pulsed T2 cells in an HLA-A*0201-restricted manner.

Functional avidity of Lengsin-specific CTL clones. To evaluate the functional avidity of Lengsin(206-215)- or Lengsin(270279)-specific CTL clones for cognate peptide-MHC ligands, peptide titration experiments were performed in cytotoxicity assay (Fig. 3). The peptide concentration required to obtain half-maximal lysis (EC50) by CTL clones 3C9, 1A1 and $1 \mathrm{G} 12$ was $11.0,11.2$ and $4.0 \mathrm{nM}$, respectively. These results suggest that Lengsin(206-215)-specific CTL clone 3C9 and Lengsin(270-279)-specific CTL clone 1A1 have approximately similar avidity, and Lengsin(270-279)-specific CTL clone 1G12 has relatively higher avidity than these two clones for cognate peptide-MHC ligands.

Lengsin(270-279)-specific CTL clone recognizes Lengsintransfected target cells in the absence of peptide, but Lengsin (206-215)-specific CTL clone fails. Next, we evaluated the ability of Lengsin(206-215)- or Lengsin(270-279)-specific CTL clones to recognize HLA-A $0201^{+} /$Lengsin $^{+}$target cells. These CTL clones were incubated with COS-7 cells expressing HLA-A*0201 and/or Lengsin, SK-Hep-1-mock (HLA-A*0201+ Lengsin ${ }^{-}$) and SK-Hep-1-Lengsin (HLA-A $0201^{+} /$Lengsin ${ }^{+}$) in the presence or absence of cognate peptide (Fig. 4). Specific IFN- $\gamma$ production of Lengsin(270-279)-specific CTL clones $1 \mathrm{~A} 1$ and $1 \mathrm{G} 12$ was detectable against COS-7 cells expressing both HLA-A*0201 and Lengsin, but not the non-treated, and only HLA-A*0201 or Lengsin-cDNA-transfected COS-7 cells in the absence of cognate peptide. In contrast, IFN- $\gamma$ production of Lengsin(206-215)-specific CTL clone 3C9 was not detectable against COS-7 cells expressing both HLA-A*0201 and Lengsin in the absence of cognate peptide (Fig. 4A). Furthermore, specific IFN- $\gamma$ production of Lengsin(270-279)specific CTL clones $1 \mathrm{~A} 1$ and $1 \mathrm{G} 12$ was detectable against both HLA-A*0201 and Lengsin-positive cell line SK-Hep-1-Lengsin (HLA-A $0201^{+} /$Lengsin $^{+}$), but not Lengsin-negative SK-Hep1-mock (HLA-A $0201^{+} /$Lengsin ${ }^{-}$) in the absence of cognate peptide (Fig. 4B). The specific IFN- $\gamma$ production was blocked by HLA-A2-specific mAb, but not the isotype control, suggesting that the observed production was HLA-A2 restricted (Fig. 4C). In contrast, IFN- $\gamma$ production of Lengsin(206-215)-specific CTL clone 3C9 was not detectable against SK-Hep-1-Lengsin in the absence of cognate peptide (Fig. 4B). These results suggest that the Lengsin(270-279)-specific CTL clones can specifically recognize Lengsin-transfected HLA-A* $0201^{+}$ target cells; however, the Lengsin(206-215)-specific CTL clone is unable to recognize these target cells unless Lengsin(206215 ) is exogenously added.

Lengsin(270-279)-specific CTL clone also recognizes HLA-A* $\mathrm{O2O}^{+}$lung carcinoma cells endogenously expressing Lengsin. We previously reported that Lengsin was expressed in the lung carcinoma cell line 1-87 (16). In order to investigate whether Lengsin(270-279) is naturally processed and presented on the surface of lung carcinoma cells, we generated a 1-87-A0201 (HLA-A*0201+/Lengsin ${ }^{+}$) stable cell line as a target of both HLA-A*0201 and Lengsin-positive lung carcinoma cells. Lengsin(206-215)-specific CTL clone 3C9 or Lengsin(270-279)-specific CTL clone 1G12 was incubated with 1-87-mock (HLA-A*0201/Lengsin ${ }^{+}$) or $1-87-A 0201$ (HLA-A ${ }^{*} 0201^{+} /$Lengsin ${ }^{+}$) in the presence or absence of cognate peptide. Lengsin(270-279)-specific CTL clone 1G12 was able to specifically recognize $1-87-\mathrm{A} 0201$ (HLA-A $0201^{+} /$Lengsin ${ }^{+}$) but not the non-HLA-A*0201 cell line 1-87-mock (HLA$\mathrm{A}^{*} 0201^{-} /$Lengsin $^{+}$) in the absence of cognate peptide (Fig. 5A). The specific IFN- $\gamma$ production was blocked by HLA-A2specific $\mathrm{mAb}$ but not the isotype control $\mathrm{mAb}$, suggesting that the observed IFN- $\gamma$ production was HLA-A2 restricted 
A. $3 C 9$ (Lengsin(206-215))
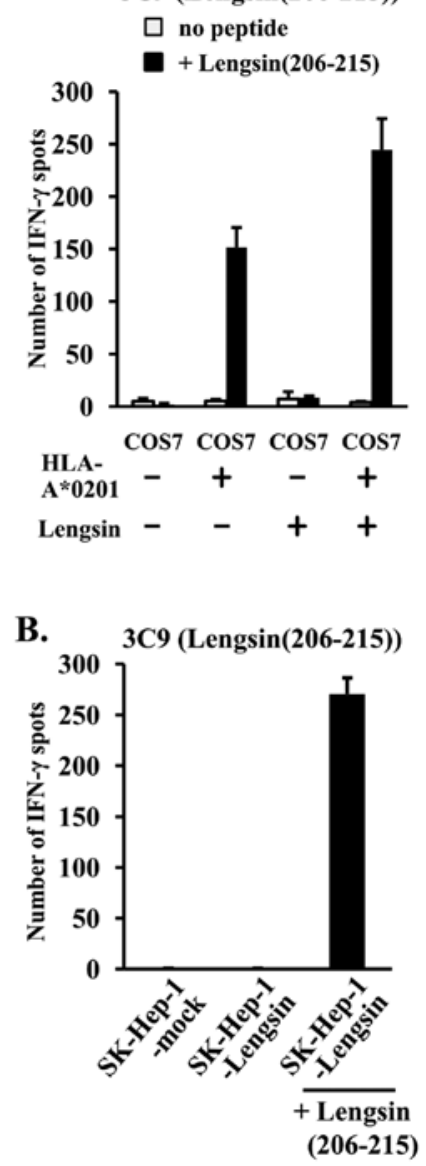

c.

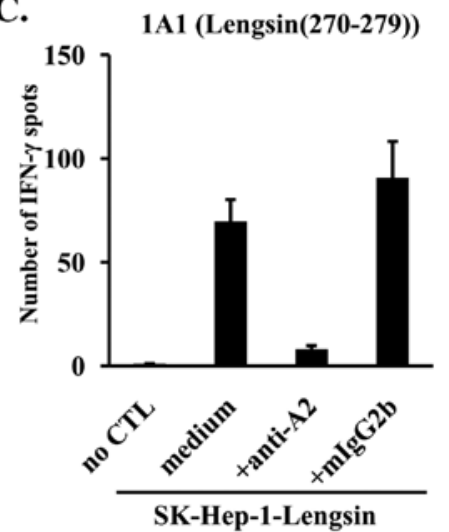

1A1 (Lengsin(270-279))

$\square$ no peptide

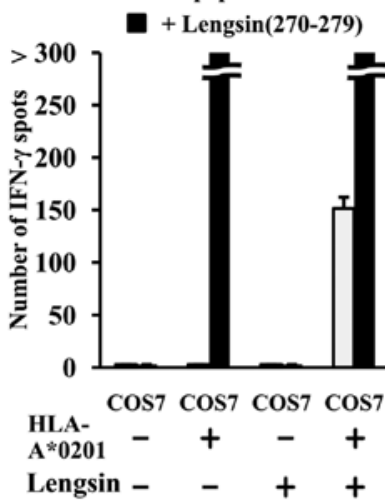

$1 \mathrm{A1}$ (Lengsin(270-279))

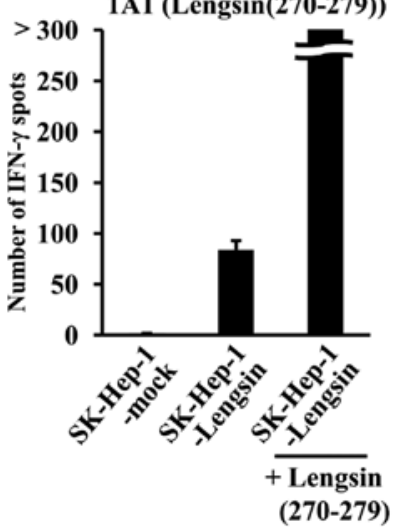

1 G12 (Lengsin(270-279))

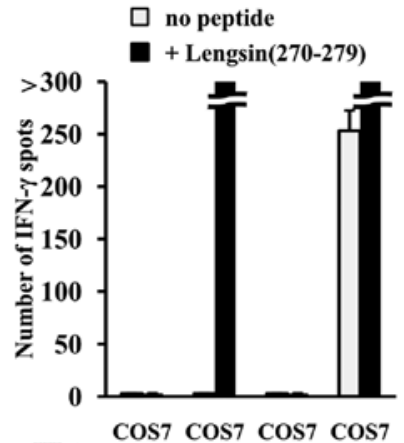

HLA- -+ -

Lengsin - -++
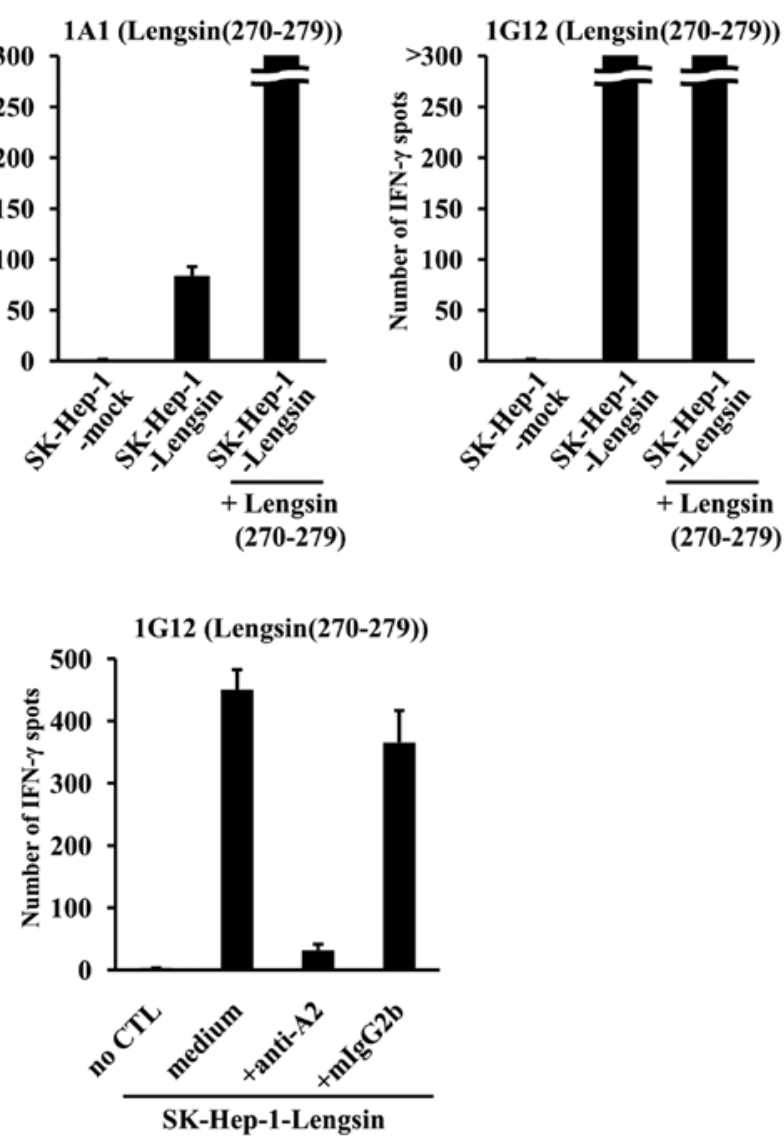

Figure 4. Recognition of Lengsin transfectant cells by Lengsin-specific CTL clones. IFN- $\gamma$-producing CTLs were detected by IFN- $\gamma$ ELISPOT. (A) COS-7 cells were transiently transfected with plasmids encoding HLA-A*0201 and/or Lengsin. Forty-eight hours after transfection, Lengsin(206-215)-specific CTL

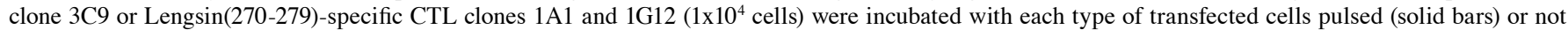
(shaded bars) for $1 \mathrm{~h}$ with $1 \mu \mathrm{M}$ indicated peptides. (B) Lengsin(206-215)-specific CTL clone 3C9 or Lengsin(270-279)-specific CTL clones 1A1 and 1G12 were incubated with SK-Hep-1-mock (HLA-A*0201 $/$ Lengsin-) or SK-Hep-1-Lengsin (HLA-A*0201 $/$ Lengsin ${ }^{+}$) pulsed or not for $1 \mathrm{~h}$ with $1 \mu \mathrm{M}$ indicated peptides. (C) Blocking experiments were performed using HLA-A2-specific mAb (BB7.2) or isotype control mAb (mIgG2b). Lengsin(270-279)-specific CTL clones 1A1 and $1 \mathrm{G} 12\left(1 \times 10^{4}\right.$ cells) were incubated with SK-Hep-1-Lengsin $\left(\mathrm{HLA}-\mathrm{A}^{*} 0201^{+} /\right.$Lengsin $\left.{ }^{+}\right)$as stimulator cells. Results are expressed as the mean \pm SD.

(Fig. 5B). In addition, Lengsin(270-279)-specific CTL clone $1 \mathrm{G} 12$ was able to specifically lyse 1-87-A0201 (HLA-A*0201 ${ }^{+}$ Lengsin ${ }^{+}$(Fig. 5C). In contrast, Lengsin(206-215)-specific CTL clone 3C9 was unable to recognize and lyse 1-87-A0201 (HLA-A ${ }^{*} 0201^{+} /$Lengsin ${ }^{+}$) in the absence of cognate peptide (Fig. 5A and C). These results suggest that the Lengsin(270279)-specific CTL clone can recognize the HLA-A*0201+ lung carcinoma cell line endogenously expressing Lengsin in an
HLA-A*0201-restricted fashion; therefore, Lengsin(270-279) is naturally processed and presented by HLA-A*0201 molecules on the surface of lung carcinoma cells expressing Lengsin.

\section{Discussion}

In order to demonstrate that predicted candidate peptides are naturally presented peptides on tumor cells, it is necessary that 
A.

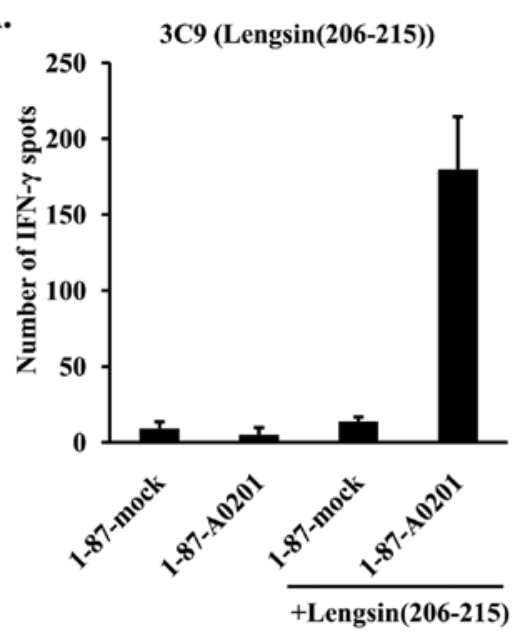

B.

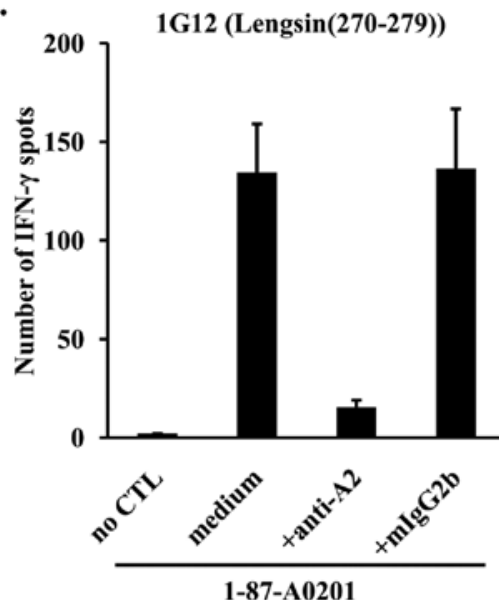

$1 G 12$ (Lengsin(270-279))

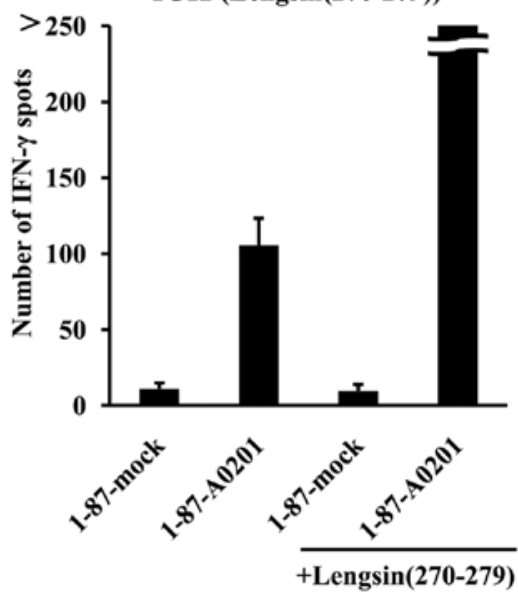

C.
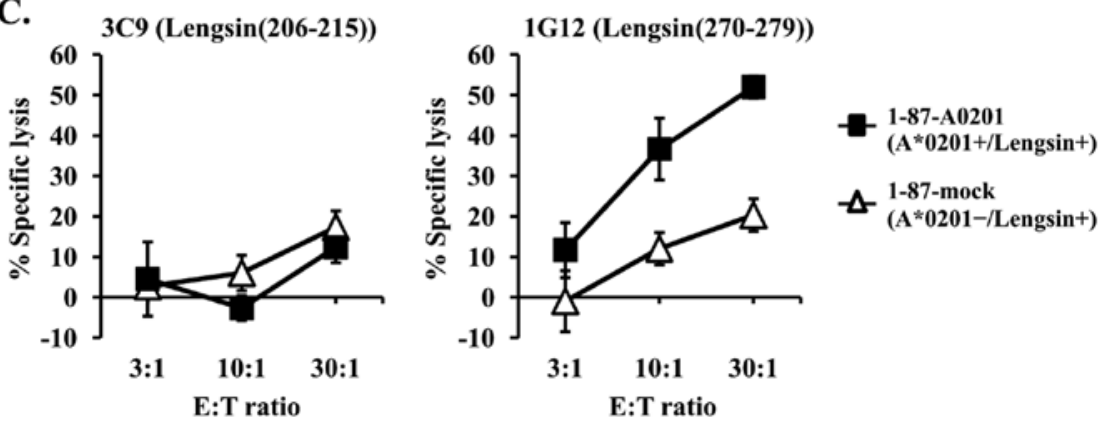

Figure 5. Recognition of lung carcinoma cells endogenously expressing Lengsin by Lengsin-specific CTL clones. (A) Lengsin(206-215)-specific CTL clone 3 C9 or Lengsin(270-279)-specific CTL clone $1 \mathrm{G} 12\left(5 \times 10^{5}\right.$ cells) was incubated with 1-87-mock (HLA-A*0201\%/Lengsin $\left.{ }^{+}\right)$or $1-87-A 0201$ (HLA-A*0201 ${ }^{+} /$ Lengsin ${ }^{+}$) pulsed or not for $1 \mathrm{~h}$ with $1 \mu \mathrm{M}$ indicated peptides. IFN- $\gamma$-producing CTLs were detected by IFN- $\gamma$ ELISPOT. (B) Blocking experiments were performed using HLA-A2-specific mAb (BB7.2) or isotype control mAb (mIgG2b). Lengsin(270-279)-specific CTL clone 1G12 (5x105 cells) was incubated with 1-87-A0201 (HLA-A*0201/Lengsin ${ }^{+}$) as stimulator cells. IFN- $\gamma$-producing CTLs were detected by IFN- $\gamma$ ELISPOT. (C) Specific lysis of lung carcinoma cell lines 1-87-mock (HLA-A*0201//Lengsin ${ }^{+}, \Delta$ ) and 1-87-A0201 (HLA-A*0201+/Lengsin ${ }^{+}$, $\bullet$ ) by Lengsin(206-215)-specific CTL clone 3C9 or Lengsin (270-279)-specific CTL clone 1G12. Results are expressed as the mean \pm SD.

peptide-specific CTL clones or lines are induced by the peptides and the CTL clones or lines specifically recognize the tumor cells. In this study, Lengsin(206-215) and Lengsin(270-279) were able to induce peptide-specific CTLs in HHD mice and humans, indicating that these peptides could be immunogenic. Especially, we were able to establish each peptide-specific CTL clone from human PBMCs. The Lengsin(270-279)-specific CTL clones specifically recognized cognate peptide-pulsed T2 cells and HLA-A*0201+ tumor cells endogenously expressing
Lengsin, suggesting that Lengsin(270-279) is naturally processed and presented on the surface of Lengsin-expressing tumor cells in association with HLA-A*0201. Therefore, Lengsin(270-279) may be a new target for antigen-specific T-cell immunotherapy against lung cancer. However, the Lengsin(206-215)-specific CTL clone failed to recognize those tumor cells expressing Lengsin, although it showed efficient recognition against $\mathrm{HLA}-\mathrm{A}^{*} 0201^{+}$target cells in the presence of exogenously added peptide. 
CTLs induced by reverse immunology approaches often fail to recognize HLA-matched tumor targets expressing specific genes (24-27). Possible explanations with respect to each factor of the induced CTLs and the targeted tumor cells seem plausible.

With regard to the factor of induced CTLs, they might not have sufficient avidity to recognize a limited number of naturally presented peptides on the surface of tumor cells (28-30). Consequently, it is necessary to induce high-avidity tumorreactive CTLs. Reported methods for inducing high-avidity CTLs include stimulation with low concentration of peptides $(31,32)$, with three costimulatory molecules (B7-1, ICAM-1 and LFA-3) (33), cultured with IL-12 (34) or IL-15 (35), and using allogeneic PBMCs as a source of CTLs (36-38). Additionally, it is very important to demonstrate recognition of tumor cells by CTL clones but not bulk CTL populations in terms of specificity. The bulk CTL populations that were used may have contained distinct CTL clones, some of which were responsible for peptide recognition and others that accounted for the apparent tumor reactivity (39). In fact, it has been reported that hTERT(540-548) is controversial as to its status as a naturally processed and presented peptide due to different results depending on assessment of bulk CTL populations $(11,40)$ or CTL clones $(41-43)$. Therefore, it is also important to establish highly avid tumor-reactive CTL clones for evaluation of specific tumor reactivity of CTLs. Melanoma-associated antigen-specific high-avidity tumor-reactive CTL clones have been generated by CD107a-guided sorting (44). In our laboratory, we recently generated GPC3(144-152)-specific high-avidity tumor-reactive CTL clones by CD107a-guided sorting from PBMCs of patients vaccinated with GPC3(144-152) peptide (45). In this study, we evaluated tumor reactivity using CTL clones, but not bulk CTL populations. Lengsin(206-215)specific high-avidity tumor-reactive CTL clones could possibly be induced and generated by further studies using the above methods.

With regard to the factor of targeted tumor cells, the predicted candidate peptides might not be processed and presented on the surface of tumor cells. In this study, Lengsin(206-215)specific CTL clone 3C9 and Lengsin(270-279)-specific CTL clone 1A1 showed approximately similar avidity in peptide titration experiments. CTL clone $1 \mathrm{~A} 1$ recognized target cells expressing Lengsin; however, CTL clone 3C9 failed. For this reason, Lengsin(206-215) might not be processed and presented on target cells. Peptides presented by MHC class I molecules are usually derived from intracellular proteins that are degraded by the proteasome (46). In contrast, by using the proteasome inhibitor lactacystin, it has happened that the expression of naturally processed peptide is sharply increased by a proteasome-independent mechanism, resulting in enhanced recognition by CTL clones $(25,47-49)$. It would be interesting to examine whether Lengsin(206-215)- or Lengsin(270-279)-specific CTL clones recognize proteasome inhibitor-treated tumor cells.

As a CTL-independent approach, tandem mass spectrometry (MS) can provide direct identification of naturally presented peptides eluted from MHC class I molecules (50-52). Using tandem MS analysis, we can positively demonstrate whether Lengsin(206-215) or Lengsin(270-279) are naturally presented peptides on the surface of tumor cells. In addi- tion, a new presented peptide derived from Lengsin might be detected.

\section{Acknowledgements}

This work was supported in part by a Grant-in-Aid for Clinical Research from the Ministry of Health, Labour and Welfare, Japan. Human HLA-A*02011 cDNA (RDB no. 2866) was provided by RIKEN BRC through the National Bio-Resources Project of MEXT, Japan.

\section{References}

1. Jemal A, Bray F, Center MM, Ferlay J, Ward E and Forman D: Global cancer statistics. CA Cancer J Clin 61: 69-90, 2011.

2. Morgan RA, Dudley ME, Wunderlich JR, et al: Cancer regression in patients after transfer of genetically engineered lymphocytes. Science 314: 126-129, 2006.

3. Johnson LA, Morgan RA, Dudley ME, et al: Gene therapy with human and mouse T-cell receptors mediates cancer regression and targets normal tissues expressing cognate antigen. Blood 114: 535-546, 2009.

4. Robbins PF, Morgan RA, Feldman SA, et al: Tumor regression in patients with metastatic synovial cell sarcoma and melanoma using genetically engineered lymphocytes reactive with NY-ESO-1. J Clin Oncol 29: 917-924, 2011.

5. Browning M and Krausa P: Genetic diversity of HLA-A2: evolutionary and functional significance. Immunol Today 17: 165-170, 1996.

6. Viatte S, Alves PM and Romero P: Reverse immunology approach for the identification of CD8 T-cell-defined antigens: advantages and hurdles. Immunol Cell Biol 84: 318-330, 2006.

7. van der Bruggen P, Bastin J, Gajewski T, et al: A peptide encoded by human gene MAGE-3 and presented by HLA-A 2 induces cytolytic T lymphocytes that recognize tumor cells expressing MAGE-3. Eur J Immunol 24: 3038-3043, 1994

8. Celis E, Tsai V, Crimi C, et al: Induction of anti-tumor cytotoxic T lymphocytes in normal humans using primary cultures and synthetic peptide epitopes. Proc Natl Acad Sci USA 91: 2105-2109, 1994.

9. Pascolo S, Bervas N, Ure JM, Smith AG, Lemonnier FA and Perarnau B: HLA-A2.1-restricted education and cytolytic activity of CD8(+) T lymphocytes from beta2 microglobulin (beta2m) HLA-A2.1 monochain transgenic $\mathrm{H}-2 \mathrm{Db}$ beta2 $\mathrm{m}$ double knockout mice. J Exp Med 185: 2043-2051, 1997.

10. Firat H, Garcia-Pons F, Tourdot S, et al: H-2 class I knockout, HLA-A2.1-transgenic mice: a versatile animal model for preclinical evaluation of antitumor immunotherapeutic strategies. Eur J Immunol 29: 3112-3121, 1999.

11. Minev B, Hipp J, Firat H, Schmidt JD, Langlade-Demoyen P and Zanetti M: Cytotoxic T cell immunity against telomerase reverse transcriptase in humans. Proc Natl Acad Sci USA 97: 4796-4801, 2000.

12. Komori H, Nakatsura T, Senju S, et al: Identification of HLA-A2or HLA-A24-restricted CTL epitopes possibly useful for glypican-3-specific immunotherapy of hepatocellular carcinoma. Clin Cancer Res 12: 2689-2697, 2006.

13. Passoni L, Scardino A, Bertazzoli C, et al: ALK as a novel lymphoma-associated tumor antigen: identification of 2 HLA-A2.1restricted CD8 ${ }^{+}$T-cell epitopes. Blood 99: 2100-2106, 2002.

14. Hassainya Y, Garcia-Pons F, Kratzer R, et al: Identification of naturally processed HLA-A2--restricted proinsulin epitopes by reverse immunology. Diabetes 54: 2053-2059, 2005.

15. Alves PM, Faure O, Graff-Dubois S, et al: EphA2 as target of anticancer immunotherapy: identification of HLA-A*201restricted epitopes. Cancer Res 63: 8476-8480, 2003.

16. Nakatsugawa M, Hirohashi Y, Torigoe T, et al: Novel spliced form of a lens protein as a novel lung cancer antigen, Lengsin splicing variant 4. Cancer Sci 100: 1485-1493, 2009.

17. Lang RA: Apoptosis in mammalian eye development: lens morphogenesis, vascular regression and immune privilege. Cell Death Differ 4: 12-20, 1997.

18. Martin WD, Egan RM, Stevens JL and Woodward JG: Lensspecific expression of a major histocompatibility complex class I molecule disrupts normal lens development and induces cataracts in transgenic mice. Invest Ophthalmol Vis Sci 36: 1144-1154, 1995. 
19. Akatsuka Y, Goldberg TA, Kondo E, et al: Efficient cloning and expression of HLA class I cDNA in human B-lymphoblastoid cell lines. Tissue Antigens 59: 502-511, 2002.

20. Hirohashi Y, Torigoe T, Maeda A, et al: An HLA-A24-restricted cytotoxic T lymphocyte epitope of a tumor-associated protein, survivin. Clin Cancer Res 8: 1731-1739, 2002.

21. Tsukahara T,Kawaguchi S, Torigoe T, et al: HLA-A*0201-restricted CTL epitope of a novel osteosarcoma antigen, papilloma-virus binding factor. J Transl Med 7: 44, 2009.

22. Wang XM, Terasaki PI, Rankin GW Jr, Chia D, Zhong HP and Hardy S: A new microcellular cytotoxicity test based on calcein AM release. Hum Immunol 37: 264-270, 1993.

23. Kondo M, Sakuta K, Noguchi A, et al: Zoledronate facilitates large-scale ex vivo expansion of functional gammadelta $\mathrm{T}$ cells from cancer patients for use in adoptive immunotherapy. Cytotherapy 10: 842-856, 2008.

24. Tanzarella S, Fleischhauer K, van Endert P, Bordignon C and Traversari C: Characterization of antigenic peptide epitopes by reverse immunology: induction of cytotoxic T lymphocytes specific for exogenous peptide only. Int J Cancer 72: 912-915, 1997.

25. Noppen C, Levy F, Burri L, et al: Naturally processed and concealed HLA-A2.1-restricted epitopes from tumor-associated antigen tyrosinase-related protein-2. Int J Cancer 87: 241-246, 2000.

26. So T, Hanagiri T, Chapiro J, et al: Lack of tumor recognition by cytolytic T lymphocyte clones recognizing peptide 195-203 encoded by gene MAGE-A3 and presented by HLA-A24 molecules. Cancer Immunol Immunother 56: 259-269, 2007.

27. Parkhurst MR, Fitzgerald EB, Southwood S, Sette A, Rosenberg SA and Kawakami Y: Identification of a shared HLA-A*0201-restricted T-cell epitope from the melanoma antigen tyrosinase-related protein 2 (TRP2). Cancer Res 58: 4895-4901, 1998.

28. Yee C, Savage PA, Lee PP, Davis MM and Greenberg PD: Isolation of high avidity melanoma-reactive CTL from heterogeneous populations using peptide-MHC tetramers. J Immunol 162: 2227-2234, 1999 .

29. Karbach J, Gnjatic S, Pauligk C, et al: Tumor-reactive CD8 T-cell clones in patients after NY-ESO-1 peptide vaccination. Int J Cancer 121: 2042-2048, 2007.

30. Wilde S, Sommermeyer D, Frankenberger B, et al: Dendritic cells pulsed with RNA encoding allogeneic MHC and antigen induce $\mathrm{T}$ cells with superior antitumor activity and higher TCR functional avidity. Blood 114: 2131-2139, 2009

31. Alexander-Miller MA, Leggatt GR and Berzofsky JA: Selective expansion of high- or low-avidity cytotoxic T lymphocytes and efficacy for adoptive immunotherapy. Proc Natl Acad Sci USA 93: 4102-4107, 1996

32. Zeh HJ 3rd, Perry-Lalley D, Dudley ME, Rosenberg SA and Yang JC: High avidity CTLs for two self-antigens demonstrate superior in vitro and in vivo antitumor efficacy. J Immunol 162: 989-994, 1999

33. Hodge JW, Chakraborty M, Kudo-Saito C, Garnett CT and Schlom J: Multiple costimulatory modalities enhance CTL avidity. J Immunol 174: 5994-6004, 2005.

34. Xu S, Koski GK, Faries M, et al: Rapid high efficiency sensitization of $\mathrm{CD}^{+} \mathrm{T}$ cells to tumor antigens by dendritic cells leads to enhanced functional avidity and direct tumor recognition through an IL-12-dependent mechanism. J Immunol 171: 2251-2261, 2003.

35. Oh S, Perera LP, Burke DS, Waldmann TA and Berzofsky JA: IL-15/IL-15Ralpha-mediated avidity maturation of memory $\mathrm{CD}^{+}$T cells. Proc Natl Acad Sci USA 101: 15154-15159, 2004.
36. Sadovnikova E, Jopling LA, Soo KS and Stauss HJ: Generation of human tumor-reactive cytotoxic $\mathrm{T}$ cells against peptides presented by non-self HLA class I molecules. Eur J Immunol 28: 193-200, 1998.

37. Savage P, Gao L, Vento K, et al: Use of B cell-bound HLA-A2 class I monomers to generate high-avidity, allo-restricted CTLs against the leukemia-associated protein Wilms tumor antigen. Blood 103: 4613-4615, 2004.

38. Kronig H, Hofer K, Conrad H, et al: Allorestricted T lymphocytes with a high avidity T-cell receptor towards NY-ESO-1 have potent anti-tumor activity. Int J Cancer 125: 649-655, 2009.

39. Parkhurst MR, Riley JP, Igarashi T, Li Y, Robbins PF and Rosenberg SA: Immunization of patients with the hTERT:540548 peptide induces peptide-reactive T lymphocytes that do not recognize tumors endogenously expressing telomerase. Clin Cancer Res 10: 4688-4698, 2004.

40. Vonderheide RH, Hahn WC, Schultze JL and Nadler LM: The telomerase catalytic subunit is a widely expressed tumor-associated antigen recognized by cytotoxic T lymphocytes. Immunity 10: 673-679, 1999

41. Ayyoub M, Migliaccio M, Guillaume P, et al: Lack of tumor recognition by hTERT peptide 540-548-specific CD8(+) T cells from melanoma patients reveals inefficient antigen processing. Eur J Immunol 31: 2642-2651, 2001.

42. Purbhoo MA, Li Y, Sutton DH, et al: The HLA-A*0201-restricted hTERT(540-548) peptide is not detected on tumor cells by a CTL clone or a high-affinity T-cell receptor. Mol Cancer Ther 6: 2081-2091, 2007.

43. Chen DY, Vance BA, Thompson LB, Domchek SM and Vonderheide RH: Differential lysis of tumors by polyclonal T cell lines and T cell clones specific for hTERT. Cancer Biol Ther 6: 1991-1996, 2007.

44. Rubio V, Stuge TB, Singh N, Betts MR, Weber JS, Roederer M and Lee PP: Ex vivo identification, isolation and analysis of tumor-cytolytic T cells. Nat Med 9: 1377-1382, 2003.

45. Yoshikawa T, Nakatsugawa M and Suzuki S: HLA-A2-restricted glypican-3 peptide-specific CTL clones induced by peptide vaccine show high avidity and antigen-specific killing activity against tumor cells. Cancer Sci 102: 918-925, 2011.

46. Rock KL and Goldberg AL: Degradation of cell proteins and the generation of MHC class I-presented peptides. Annu Rev Immunol 17: 739-779, 1999.

47. Wherry EJ, Golovina TN, Morrison SE, Sinnathamby G, McElhaugh MJ, Shockey DC and Eisenlohr LC: Re-evaluating the generation of a 'proteasome-independent' MHC class I-restricted CD8 T cell epitope. J Immunol 176: 2249-2261, 2006.

48. Valmori D, Gileadi U, Servis C, et al: Modulation of proteasomal activity required for the generation of a cytotoxic T lymphocytedefined peptide derived from the tumor antigen MAGE-3. J Exp Med 189: 895-906, 1999.

49. Luckey CJ, King GM, Marto JA, et al: Proteasomes can either generate or destroy MHC class I epitopes: evidence for nonproteasomal epitope generation in the cytosol. J Immunol 161: 112-121, 1998.

50. Cox AL, Skipper J, Chen Y, et al: Identification of a peptide recognized by five melanoma-specific human cytotoxic $\mathrm{T}$ cell lines. Science 264: 716-719, 1994.

51. Schirle M, Keilholz W, Weber B, et al: Identification of tumorassociated MHC class I ligands by a novel T cell-independent approach. Eur J Immunol 30: 2216-2225, 2000.

52. Hirano N, Butler MO, Xia Z, et al: Identification of an immunogenic $\mathrm{CD}^{+} \mathrm{T}$-cell epitope derived from gamma-globin, a putative tumor-associated antigen for juvenile myelomonocytic leukemia. Blood 108: 2662-2668, 2006. 